\title{
Calidad de vida en pacientes con Enfermedad Pulmonar Obstructiva
}

\author{
Quality of life in patients with chronic obstructive pulmonary disease
}

\begin{abstract}
Sonia Elena Pineda-Higuita1*, Vidal de Jesús Ramos-Melchor², Daniela Cadavid-Carmona ${ }^{3}$
1 Bacterióloga y Laboratorista Clínica. Esp. en Ciencias Forenses, Magíster en Salud Pública. Profesora investigadora Facultad de Terapia Respiratoria y Grupo de Investigación en Salud y Comunidad -GISCo de la Fundación Universitaria Autónoma de las Américas. Medellín, Colombia. e-mail: soniapi05@yahoo.es

2 Médico. Empresa Social del Estado -E.S.E. METROSALUD. Medellín, Colombia. e-mail: vidaldej@une.net.co

3 Profesora investigadora Facultad de Terapia Respiratoria y Grupo de Investigación en Salud y Comunidad -GISCo de la Fundación Universitaria Autónoma de las Américas. Medellín, Colombia. e-mail: danycadavid@hotmail.com
\end{abstract}

Fecha de recepción: Agosto 5 - $2015 \quad$ Fecha de revisión: Junio 17 - $2016 \quad$ Fecha de aceptación: Diciembre 12 - 2016

Pineda-Higuita SE, Ramos-Melchor VdJ, Cadavid-Carmona D. Calidad de vida en pacientes con Enfermedad Pulmonar Obstructiva, Medellín. 2014. Univ. Salud. 2016;18(3):482-493. DOI: http://dx.doi.org/10.22267/rus.161803.53

\begin{abstract}
Resumen
Objetivo: Describir la calidad de vida de pacientes con enfermedad pulmonar obstructiva crónica año 2014. Materiales y Métodos: Se realizó un estudio observacional descriptivo de corte transversal para evaluar la calidad de vida de los pacientes mayores de 65 años con diagnóstico de Enfermedad Pulmonar Obstructiva Crónica. La muestra correspondió a 179 usuarios de la ESE Metrosalud (Medellín), que aceptaron participar voluntariamente en el estudio. Se aplicó una encuesta estructurada que incluyó variables sociodemográficas, algunos determinantes ambientales, sociales y aspectos relacionados con la enfermedad. Igualmente se incluyó la escala de calidad de vida Saint George la cual está constituida por tres dimensiones: síntomas, actividades e impacto. Resultados: Los pacientes encuestados tenían entre 65 y 99 años de edad, al evaluar la calidad de vida con el cuestionario Saint George la dimensión más impactada fue la actividad; se obtuvo asociación estadísticamente significativa entre calidad de vida total y el nivel de escolaridad $(\mathrm{P}=0,005)$, etnia $(\mathrm{P}=0,004)$, y algunas variables relacionadas con la enfermedad. Conclusiones: Existe una asociación entre la calidad relacionada con la salud, según la evaluación del SGRQ y el grado de la enfermedad.
\end{abstract}

Palabras clave: Calidad de vida; signos y síntomas; Enfermedad Pulmonar Obstructiva Crónica; impacto en el estado de salud; oxigenoterapia. (Fuente: DeCS, Bireme).

\begin{abstract}
Objective: To describe the quality of life of patients with chronic obstructive pulmonary disease 2014. Materials and Methods: A descriptive observational cross-sectional study was performed to evaluate the quality of life of patients older than 65 and who had a diagnosis of chronic obstructive pulmonary disease. The sample corresponded to 179 users of ESE Metrosalud (Medellín), who agreed to participate voluntarily in the study. A structured survey that included socio-demographic variables, some environmental and social determinants, and aspects related to the disease was applied. Besides, the Saint George quality of life scale was also included, which is composed of three dimensions: symptoms, activities and impact. Results: The surveyed patients were between 65 and 99 years old, when they took the Saint George quality of life questionnaire, the most impacted dimension was the activity; it was obtained a statistically significant association between total quality of life and the level of schooling $(\mathrm{P}=0.005)$, ethnic group
\end{abstract}


$(\mathrm{P}=0.004)$, and some variables related with the disease. Conclusions: There is an association between the quality related with health, according to the evaluation of the SGRQ and the extent of the disease.

Keywords: Quality of life; signs and symptoms; Pulmonary Disease, Chronic Obstructive; health evaluation; oxygen therapy. (Source: DeCS, Bireme).

\section{Introducción}

La enfermedad pulmonar obstructiva crónica (EPOC), es una enfermedad respiratoria, generalmente irreversible, caracterizada por la existencia de una obstrucción progresiva de las vías aéreas, causada principalmente por el humo del tabaco. Su síntoma principal es una disminución de la capacidad respiratoria que avanza lentamente con el paso de los años y ocasiona un deterioro considerable en la calidad de vida de las personas afectadas ${ }^{1}$.

En el 2007, el estudio de prevalencia de EPOC en Colombia (PREPOCOL), realizado en cinco ciudades, determinó que a nivel nacional 9 de cada 100 personas mayores de 40 años tenían EPOC, este porcentaje se distribuyó en $8,5 \%$ en Bogotá, $6,2 \%$ en Barranquilla, $7,9 \%$ en Bucaramanga, 8,5\% en Cali y 13,5\% en Medellín. Las diferencias de prevalencia entre las ciudades se relacionan directamente con el porcentaje de sujetos fumadores en cada ciudad, que varió entre el 14\% en Barranquilla hasta el 29\% en Medellín, y su porcentaje va en aumento principalmente en jóvenes escolares ${ }^{2}$.

Las enfermedades crónicas, como la EPOC están influenciadas no solo por aspectos de tipo fisiopatológicos, sino también por determinantes sociales, ambientales, $\mathrm{y}$ estilos de vida que conllevan a un deterioro de la misma3-5.

Los determinantes de salud se describen como las propiedades basadas en el estilo de vida, afectadas por amplias fuerzas sociales, económicas y políticas que influyen en la calidad de la salud. En las últimas décadas, las crecientes disparidades de salud en los países desarrollados y los países en vía de desarrollo han sido asociadas a estos factores ${ }^{6}$.

Según la Comisión sobre Determinantes Sociales de la Salud (CDSS), estas son las circunstancias en que las personas nacen, crecen, viven, trabajan y envejecen, incluido el sistema de salud. Esas circunstancias son el resultado de la distribución del dinero, el poder y los recursos a nivel mundial, nacional y local, que depende a su vez de las políticas adoptadas y explican la mayor parte de las inequidades en salud, esto es, de las diferencias injustas y evitables observadas en y entre los países en lo que respecta a la situación de salud?.

Las condiciones en las que viven y trabajan las personas pueden ayudar a crear o destruir su salud: falta de ingresos, vivienda inadecuada, lugares de trabajo inseguros y falta de acceso a los sistemas de salud, son algunos de los determinantes sociales de la salud que generan inequidades entre y dentro los países. En las políticas de salud han predominado las soluciones centradas en el tratamiento de las enfermedades, sin incorporar adecuadamente intervenciones sobre las "causas de las causas". En consecuencia, los problemas de salud se han mantenido al igual que las inequidades y la falta de atención oportuna. Las intervenciones se han concentrado en lo curativo y han sido insuficientes y no permitirán alcanzar las metas de salud de los Objetivos para el Milenio ${ }^{7}$.

Respecto a la morbilidad causada por factores ambientales es mucho más elevada en el mundo en desarrollo que en los países desarrollados. Según la Organización Mundial de la Salud (OMS), se estima que en 2012 perdieron la vida 12,6 millones de personas por vivir o trabajar en ambientes poco saludables: casi una cuarta parte del total mundial de muertes. Los factores de riesgo ambientales, como la contaminación del aire, agua y suelo, la exposición a productos químicos, el cambio climático y la radiación ultravioleta, contribuyen a más de 100 enfermedades o traumatismos. El 23\% de las muertes están relacionadas con el medio ambiente, esto representa los 12,6 millones de 
muertes al año y de estas 847.000 ocurren en las Américas. Las enfermedades respiratorias se encuentran dentro de las 10 principales causas de muerte relacionadas con el medio ambiente ${ }^{8}$.

Estos desequilibrios ambientales están incidiendo de manera creciente y con diversos gradientes de intensidad sobre la calidad de vida de toda la población, afectando directa e indirectamente su salud. La EPOC es un problema de salud pública de enormes proporciones que está aumentando en todo el mundo por su morbimortalidad, costos sociales y económicos. En los últimos 30 años la mortalidad mundial ha aumentado en un $163 \%$. La OMS calcula que para el año 2020 la EPOC pasará de ser la causa número 12 de enfermedad en el mundo a la quinta causa de años de vida perdidos, ajustados por invalidez, y del puesto sexto como causa más común de muerte a la tercera causa de mortalidad, sólo superada por el cáncer y las enfermedades cardiovasculares, situándose incluso por delante del SIDA 9 .

En un intento de dar respuesta a valoraciones más amplias de la salud, surge a mediados de los años setenta, el término "calidad de vida", que aparece como un concepto multidimensional que valora el efecto que la enfermedad tiene sobre el individuo en su contexto individual, familiar y social ${ }^{10}$.

La evaluación de la calidad de vida en personas con enfermedades respiratorias se constituye en una oportunidad para conocer el impacto real de la enfermedad sobre la vida diaria. Por otra parte, es necesario tener en cuenta que si bien lo fisiológico tiene gran afectación, existen otros aspectos tales como el ambiente y el entorno social que influyen en la calidad de vida de estas personas y a medida que la enfermedad progresa, los pacientes con EPOC experimentan una disminución de la Calidad de Vida Relacionada con la Salud (CVRS) y aumento en el deterioro de su capacidad para trabajar y participar en actividades físicas y sociales ${ }^{12}$.

En la mayoría de estudios, los efectos de la enfermedad sobre el paciente y el de las terapias sobre la EPOC se evalúan por medio de pruebas fisiológicas; sin embargo, estas no tienen en cuenta la percepción del individuo con respecto a su enfermedad ${ }^{13}$.

Se han utilizado diversos cuestionarios para evaluar la calidad de vida, algunos realizan esta valoración de manera general y otros son considerados específicos, para el caso de la EPOC existe un cuestionario respiratorio denominado Saint George's (SGRQ), que permite valorar de forma estandarizada la impresión subjetiva que los pacientes tienen sobre su propia enfermedad; una de las principales aplicaciones de este cuestionario es la de detectar aquellos factores que más condicionan la vida diaria de los pacientes con EPOC, lo que permite incidir en determinados aspectos del tratamiento con el objetivo de mejorar su calidad de vida ${ }^{14}$.

Teniendo en cuenta lo anterior, se justifica el interés de describir la calidad de vida de los pacientes con EPOC de la E.S.E. Metrosalud de la ciudad de Medellín y poder contribuir a mejorar el impacto de los programas de intervención en pacientes con esta enfermedad.

\section{Materiales y métodos}

\section{Diseño del estudio}

Se realizó un estudio observacional descriptivo de corte transversal, el cual abordó algunas características que conforman la calidad de vida de los pacientes mayores de 65 años diagnosticados con EPOC.

\section{Tamaño muestral}

La población estuvo conformada por 615 personas, las cuales se encontraban activas al momento de realizar el estudio. Para determinar el tamaño de la muestra se tuvo en cuenta el total de pacientes, un nivel de confianza del 95\% y un error de estimación del 5\%. De lo anterior se obtuvo un tamaño muestral de 179 usuarios los cuales fueron seleccionados mediante un muestreo aleatorio simple. Los pacientes fueron contactados vía telefónica; quienes aceptaron participar, se entrevistaron en sus residencias, se les explicaron los objetivos de la investigación, posteriormente firmaron el consentimiento informado y diligenciaron una encuesta 
estructurada. Se realizó la prueba piloto a 10 personas, pero no se incluyeron esos pacientes en la evaluación de la muestra final.

\section{Procedimientos}

Se utilizó fuente de información primaria basada en la encuesta estructurada de aplicación individual que incluyó variables sociodemográficas, algunos determinantes ambientales, sociales y aspectos relacionados con la enfermedad. Igualmente se incluyó la escala de calidad de vida Saint George el cual consta de tres dimensiones: síntomas (presentados debido a la patología pulmonar), actividades (circunstancias que causan o están limitadas por la disnea) e impacto(funcionamiento social y psicológico que pueden alterar el estilo de vida del paciente), para un total de 50 preguntas donde 10 son de opción múltiple y 40 de verdadero o falso, este instrumento permite valorar la calidad de vida de 0-100 donde las puntuaciones más altas indican una mayor alteración de la misma ${ }^{14}$.

Los criterios de inclusión fueron pacientes con EPOC en la ESE Metrosalud de Medellín, mayores de 65 años, que no estuvieran hospitalizados y que aceptaran libremente participar en el estudio. El cuestionario previamente estandarizado se aplicó a manera de entrevista por los investigadores principales $\mathrm{y}$ dos terapeutas respiratorios, quienes fueron capacitadas oportunamente.

\section{Consideraciones éticas}

El proyecto fue aprobado por el Comité de ética institucional de la E.S.E Metrosalud (Acta 082012). Todos los pacientes firmaron el Consentimiento Informado cumpliendo con las normas de la Resolución 008430 de 1993 del Ministerio de Salud de Colombia y la declaración de Helsinki de 1975. Los datos encontrados fueron de uso exclusivo del grupo de investigadores y se guardaran por 5 años en la dirección de Investigaciones, cumpliendo así el principio de confidencialidad.

\section{Análisis Estadístico}

Se construyó una base de datos en ACCES, la cual fue tratada y depurada en el paquete estadístico PRISMA (licencia de la Fundación Universitaria Autónoma de las Américas). Se realizó un análisis descriptivo y un análisis bivariado con todas las variables sociodemográficas, determinantes ambientales, sociales y aspectos relacionados con la enfermedad y cada una de las dimensiones de la escala de calidad de vida Saint George. Se realizó una descripción individual de las variables del estudio a través de distribuciones de frecuencia para las variables cualitativas $\mathrm{y}$ medidas de posición y tendencia central para las cuantitativas.

Las variables de especial interés para el estudio se presentaron en tablas según la naturaleza de la misma y el número de categorías, en el caso de las cualitativas, se aplicaron pruebas de $\mathrm{Chi}^{2}$ o test exacto de Fisher $y$ en el caso de datos cuantitativos la $\mathrm{t}$ de Student, prueba de normalidad y las pruebas no paramétricas (U de Mann-Whitney y K de Kruskal-Wallis) cuando fue necesario. Se procedió al cruce de variables que respondían a los objetivos específicos de la investigación para determinar la asociación estadística entre las mismas, donde se tomaron como variables dependientes la calidad de vida con sus respectivas dimensiones: síntomas, actividades e impacto y los determinantes sociales, ambientales y aspectos relacionadas con la enfermedad como variables independientes.

Posteriormente, para identificar los determinantes ambientales y sociales que afectan la calidad de vida de pacientes y los relacionados con la EPOC, se realizó un modelo de regresión logística que permitió identificar asociaciones y la influencia de algunas dimensiones en la variabilidad de la calidad de vida y los determinantes ambientales, sociales y los relacionados con la enfermedad por medio de ORs crudos y ajustados (Variables dependientes: calidad de vida y dimensiones de la escala Saint George), las dimensiones fueron recodificadas como variable Dummy $(0=$ mínimo o bajo riesgo $1=$ alto o máximo riesgo, de acuerdo a cada dimensión expuesta). 


\section{Resultados}

\section{Características sociodemográficas}

Se encuestaron un total de 179 pacientes mayores de 65 años diagnosticados con EPOC de la E.S.E Metrosalud con edades entre 65 y 99 años, donde aproximadamente el $50 \%$ de las pacientes tenían 76 años o menos, y un promedio de edad de 77,08 $( \pm 7,979)$ años; el $44,7 \%$ refirió haber estudiado la primaria y solo el 10,6\% secundaria. El 42,5\% reportaron estar viudos, el $27,9 \%$ estaban casados, el $17,9 \%$ solteros y el $10,6 \%$ se encontraban divorciados. El 50,8\% viven con sus hijos, el $38,5 \%$ con sus familiares y el porcentaje restante con otras personas.
El $82,7 \%$ había consumido cigarrillo, el 31,3\% consumió 20 cigarrillos al día, el 13,4\% se fumaban 40 cigarrillos al día, el 19,6\% de los pacientes comenzaron a fumar a la edad de 15 años; actualmente el 20,7\% fuma; en su gran mayoría correspondían a un estrato socioeconómico bajo (Tabla 1).

\section{Calidad de vida general}

El 54,7\% de los encuestados manifestaron tener una alta alteración de la calidad de vida siendo los hombres los más afectados. El 55,34\% de las mujeres consumían cigarrillo y presentaban una alta alteración de su calidad de vida.

Tabla 1. Distribución porcentual de adultos mayores pertenecientes a la ESE Metrosalud, con enfermedad pulmonar obstructiva crónica (EPOC) de acuerdo a la alteración de calidad de vida según variables sociodemográficas, Medellín 2014

\begin{tabular}{|c|c|c|c|c|c|c|c|c|c|c|c|}
\hline \multirow[t]{2}{*}{ Calidad de vida } & & \multicolumn{2}{|c|}{$\begin{array}{c}\text { Mínima } \\
\text { alteración }\end{array}$} & \multicolumn{2}{|c|}{$\begin{array}{c}\text { Baja } \\
\text { alteración }\end{array}$} & \multicolumn{2}{|c|}{$\begin{array}{c}\text { Alta } \\
\text { alteración }\end{array}$} & \multicolumn{2}{|c|}{$\begin{array}{c}\text { Máxima } \\
\text { alteración }\end{array}$} & \multicolumn{2}{|c|}{ Total } \\
\hline & & $\mathbf{N}$ & $\%$ & $\mathbf{N}$ & $\%$ & $\mathbf{N}$ & $\%$ & $\mathbf{N}$ & $\%$ & $\mathbf{N}$ & $\%$ \\
\hline \multirow{12}{*}{ Nivel de escolaridad } & $\begin{array}{l}\text { Unidad } \\
\text { intermedia }\end{array}$ & 2 & 1,36 & 47 & 31,97 & 81 & 55,10 & 17 & 11,56 & 147 & 100,00 \\
\hline & Centro de salud & 1 & 3,13 & 7 & 21,88 & 17 & 53,13 & 7 & 21,88 & 32 & 100,00 \\
\hline & Total & 3 & 1,68 & 54 & 30,17 & 98 & 54,75 & 24 & 13,41 & 179 & 100,00 \\
\hline & Ninguno & 0 & 0,00 & 31 & 38,75 & 40 & 50,00 & 9 & 11,25 & 80 & 100,00 \\
\hline & Primaria & 1 & 1,25 & 21 & 26,25 & 48 & 60,00 & 10 & 12,50 & 80 & 100,00 \\
\hline & Secundaria & 2 & 10,53 & 2 & 10,53 & 10 & 52,63 & 5 & 26,32 & 19 & 100,00 \\
\hline & Tecnológico & 0 & 0,00 & 0 & 0,00 & 0 & 0,00 & 0 & 0,00 & 0 & 0,00 \\
\hline & Universitaria & 0 & 0,00 & 0 & 0,00 & 0 & 0,00 & 0 & 0,00 & 0 & 0,00 \\
\hline & Sin datos & 0 & 0,00 & 0 & 0,00 & 0 & 0,00 & 0 & 0,00 & 0 & 0,00 \\
\hline & Total & 3 & 1,68 & 54 & 30,17 & 98 & 54,75 & 24 & 13,41 & 179 & 100,00 \\
\hline & 1 & 1 & 2,17 & 8 & 17,39 & 27 & 58,70 & 10 & 21,74 & 46 & 100,00 \\
\hline & 2 & 1 & 1,00 & 39 & 39,00 & 52 & 52,00 & 8 & 8,00 & 100 & 100,00 \\
\hline \multirow[t]{6}{*}{ Estrato socioeconómico } & 3 & 1 & 3,23 & 6 & 19,35 & 18 & 58,06 & 6 & 19,35 & 31 & 100,00 \\
\hline & 4 & 0 & 0,00 & 1 & 50,00 & 1 & 50,00 & 0 & 0,00 & 2 & 100,00 \\
\hline & Total & 3 & 1,68 & 54 & 30,17 & 98 & 54,75 & 24 & 13,41 & 179 & 100,00 \\
\hline & Soltero(a) & 0 & 0,00 & 7 & 21,88 & 21 & 65,63 & 4 & 12,50 & 32 & 100,00 \\
\hline & Casado(a) & 2 & 4,00 & 11 & 22,00 & 31 & 62,00 & 6 & 12,00 & 50 & 100,00 \\
\hline & Divorciado(a) & 0 & 0,00 & 9 & 47,37 & 6 & 31,58 & 4 & 21,05 & 19 & 100,00 \\
\hline \multirow[t]{4}{*}{ Estado civil } & Viudo(a) & 0 & 0,00 & 27 & 35,53 & 39 & 51,32 & 10 & 13,16 & 76 & 100,00 \\
\hline & Unión libre & 1 & 50,00 & 0 & 0,00 & 1 & 50,00 & 0 & 0,00 & 2 & 100,00 \\
\hline & Sin dato & 0 & 0,00 & 0 & 0,00 & 0 & 0,00 & 0 & 0,00 & 0 & 0,00 \\
\hline & Total & 3 & 1,68 & 54 & 30,17 & 98 & 54,75 & 24 & 13,41 & 179 & 100,00 \\
\hline \multirow{5}{*}{$\begin{array}{l}\text { Consumo de cigarrillo } \\
\text { durante la vida o } \\
\text { actualmente }\end{array}$} & $\mathrm{Si}$ & 3 & 2,03 & 42 & 28,38 & 84 & 56,76 & 19 & 12,84 & 148 & 100,00 \\
\hline & No & 0 & 0,00 & 12 & 38,71 & 14 & 45,16 & 5 & 16,13 & 31 & 100,00 \\
\hline & Total & 3 & 1,68 & 54 & 30,17 & 98 & 54,75 & 24 & 13,41 & 179 & 100,00 \\
\hline & Hijos & 2 & 2,20 & 32 & 35,16 & 41 & 45,05 & 16 & 17,58 & 91 & 100,00 \\
\hline & Familiares & 1 & 1,45 & 15 & 21,74 & 45 & 65,22 & 8 & 11,59 & 69 & 100,00 \\
\hline \multirow[t]{3}{*}{ Vive con } & Otros & 0 & 0,00 & 7 & 36,84 & 12 & 63,16 & 0 & 0,00 & 19 & 100,00 \\
\hline & Sin dato & 0 & 0,00 & 0 & 0,00 & 0 & 0,00 & 0 & 0,00 & 0 & 0,00 \\
\hline & Total & 3 & 1,68 & 54 & 30,17 & 98 & 54,75 & 24 & 13,41 & 179 & 100,00 \\
\hline
\end{tabular}




\section{Dimensiones del Saint George}

Dimensión Síntomas: esta consta de ocho preguntas y se refiere a toda la sintomatología presentada debido a la patología pulmonar; entre ellos: tos, producción de expectoración, disnea o sensación de ahogo o falta de aire y sibilancias, así como duración, frecuencia y severidad de estos. Se encontró una máxima frecuencia de síntomas respiratorios en los solteros, los entrevistados en las unidades intermedias y quienes habían sido consumidores de cigarrillo (Tabla 1).

Dimensión actividades: esta dimensión contiene 16 preguntas de opción verdadera o falsa y se refiere a las actividades que se ven limitadas debido a la disnea, donde se obtuvo una media de 76,493( $\pm 21,22)$, siendo el sexo masculino quien manifestó alta presencia de limitaciones producidas por la disnea en un $30,26 \%$ con relación al 10,68\%(11) quienes respondieron tener una baja presencia de estas limitaciones. Se encontró que presentaban más limitaciones producidas por la disnea, las personas encuestadas en los centros de salud, quienes poseían secundaria, nivel socioeconómico 1, los solteros, raza mestiza, quienes vivían con familiares diferentes a los hijos, y aquellos que habían consumido cigarrillo. Quienes habían utilizado leña para cocinar los alimentos, presentaba una alta presencia de limitaciones; el 85,71\%(6) de los que tenían la enfermedad en un estado muy grave presentaba una máxima presencia de estas limitaciones. Se halló también que el promedio de horas de uso diario de oxígeno era de 17 para los adultos que indicaron una máxima, 8 para alta y 5 para baja presencia de limitaciones producidas por las dificultades para respirar (Tabla 1).

Dimensión Impacto: consta de 26 preguntas y se refiere a otras situaciones o aspectos referentes al funcionamiento social o psicológico afectado por el o los problemas respiratorios que pueden alterar el estilo de vida del paciente. A nivel general se encontró que el $45,3 \%$ de las personas encuestadas se ven impactadas en un bajo grado debido a este tipo de situaciones. Los hombres manifestaron menos presencia de problemas de funcionamiento social y psicológico en comparación con las mujeres donde el $47,75 \%(49)$ presentaba una alta presencia de problemas de este tipo producidos por el EPOC. De total de personas encuestadas en las unidades intermedias, además de no tener escolaridad y estar residenciados en un estrato 3 manifestaron baja presencia de problemas de funcionamiento social y psicológico, quienes viven con familiares, el 53,62\%(37) percibe una alta presencia de problemas (Tabla 1 ).

\section{Caracterización de los determinantes ambientales}

Las viviendas de las personas encuestadas tenían 3 cuartos y en promedio dormían 2 personas por habitación; el 97,8\% de las viviendas de las personas se encontraban construidas en ladrillo. Todos contaban con agua, energía y saneamiento básico. El 43,6 \% refirió haber cocinado en algún momento de su vida con leña y el $67,0 \%$ cuenta con escenarios deportivos cerca de su casa y el $43,6 \%$ está muy satisfecho con el ambiente que le rodea.

\section{Caracterización de los aspectos relacionados con la enfermedad}

Del total de personas encuestadas que manifestaron tener la enfermedad en un estado muy grave, se halló que 71,43\% (5) tenía una alta alteración de su calidad de vida y quienes tenían la enfermedad en un grado leve el 52,38\% (11) tenían una baja alteración. Frente al uso de oxígeno, quienes lo utilizaban 24 horas presentaban una máxima alteración. Los pacientes que utilizan inhalador presurizado, polvo seco, oxígeno y cámara espaciadora presentaban una alta afectación de su calidad de vida en relación con quienes no utilizan este tipo de elementos. El 59,21\% (45) que no tenían la vacuna contra influenza manifestaron una alta alteración de su calidad de vida.

\section{Asociaciones entre las variables independientes y la variable dependiente calidad de vida}

\section{Determinantes ambientales y calidad de vida}

Al evaluar los determinantes ambientales y calidad de vida se encontró que el 53,85\% (42) de las personas encuestadas que utilizaron leña para 
cocinar los alimentos o actualmente la utilizan refirieron una alta alteración de su calidad de vida. Los hombres presentaron mayor afectación que las mujeres; en general las personas se encontraban satisfechas con el lugar donde viven.

\section{Determinantes ambientales y síntomas}

El cocinar con leña o haberla utilizado aumenta los síntomas respiratorios.

\section{Determinantes ambientales y actividades}

En relación a estos determinantes, se halló que quienes viven en viviendas elaboradas en ladrillo el 56,57\% (99) presentaban una máxima presencia de limitaciones y el 10,29\% una baja presencia de estas. El 56,41\% (44) de las personas que utilizaban leña para cocinar los alimentos presentaron limitaciones en esta dimensión.

\section{Determinantes ambientales e Impacto}

Según los determinantes ambientales y su relación con las limitaciones presentes en los adultos a causa de la enfermedad, se tiene que del total de aquellos que tienen acceso a los servicios públicos, tenían baja presencia de problemas de funcionamiento social y psicológico. Del total de personas que indicaron cocinar con leña, el $44,87 \%(35)$ señaló una baja presencia de problemas de funcionamiento, el 6,41\%(5) una máxima y el 5,13\%(4) una mínima. Aquellos que no utilizaron leña para cocinar los alimentos, percibieron baja presencia de problemas.

\section{Aspectos relacionados con la enfermedad y síntomas}

Del total de personas que indicaron usar inhaladores el 17,54\% (30) reporta tener una máxima frecuencia de síntomas respiratorios, quienes indicaron usar inhaladores presurizados el 39,61\% (61) tiene una alta frecuencia de síntomas respiratorios. Según la vacuna anti influenza, se tiene que del total de adultos que indicaron no tenerla el 39,47\% (30) tiene una alta frecuencia de síntomas mientras que el 11,02\% (14) del total de adultos que indico si tener la vacuna muestra una mínima presencia de síntomas respiratorios. Frente al uso del oxígeno por horas al día según la frecuencia de síntomas respiratorios, se halló que del total de adultos que indicaron usar el oxígeno 24 horas al día, el 29,69\% (19) tiene una máxima frecuencia de estos síntomas respiratorios, el $50 \%$ de los adultos que señalaron usar el oxígeno entre 8 a 11 horas presentan baja presencia de síntomas respiratorios, el 13,46\% (7) de los adultos que no lo usan tiene mínima frecuencia de síntomas respiratorios. De forma general, quien usa el oxígeno por más de 15 horas al día presenta un incremento en la frecuencia de síntomas respiratorios ubicándose a la categoría alta frecuencia de los mismos. El promedio de hora de uso de oxígeno para quienes indicaron mínima frecuencia de síntomas respiratorios fue de 9 horas al día.

\section{Aspectos relacionados con la enfermedad y actividades}

El 57,14\% (12) de los adultos con EPOC en grado leve presentaban una alta presencia de limitaciones. El 85,71\% (6) de los que tenían un estado muy grave presentaba una máxima presencia y el 25,81\% (8) presentó una baja presencia de limitaciones. Además, quienes utilizan inhalador tienen más limitaciones producidas por la disnea, que quienes no lo utilizan. Quienes utilizaban inhalador presurizado el 60,39\%(93) presentó una máxima presencia de limitaciones por disnea y aquellos que utilizan ambos inhaladores (presurizado y polvo seco) indicaron tener una alta presencia de limitaciones. El 64,62\%(84) que utilizaba cámara espaciadora presentó una máxima presencia de limitaciones. Respecto a quienes estaban vacunados contra la influenza el 49,51\%(51) presentaban una baja presencia de limitaciones, el 33,98\%(35) una alta presencia y el 4,65\% (2) mínima presencia.

\section{Aspectos relacionados con la enfermedad e impacto}

De los pacientes con EPOC en estado muy grave, el 57,14\%(4) reportan una alta presencia de problemas de funcionamiento social $y$ psicológico, a causa de esta enfermedad. El total de pacientes que utilizan inhalador en polvo seco, presentaron una baja presencia de problemas de funcionamiento, quienes no utilizaron cámara 
espaciadora también tuvieron una baja presencia de estos problemas en un, 62,79\%; respecto a la vacunación contra influenza, del total de hombres que no estaban vacunados, el $42,42 \%$ señalan una baja presencia de problemas de funcionamiento $y$ el $45,45 \%$ una alta presencia de estos factores, según los promedios de horas de uso de oxígeno al día, se tiene que los adultos que indicaron una máxima presencia de problemas de funcionamiento social y psicológico tienen un promedio de 13 horas días.

\section{Factores asociados a calidad de vida}

Se encontró asociación estadísticamente significativa (Valores $\mathrm{p}<0,05$ ) entre calidad de vida y las variables sociodemográficas como, nivel de escolaridad, barrio, etnia y las variables relacionadas con la enfermedad como, grado de la enfermedad, utiliza inhaladores, tipo de inhalador, cámara espaciadora y uso de oxígeno. Adicionalmente se halló asociación significativa entre la dimensión síntomas y el grado de la enfermedad, al igual que el uso de inhaladores y las horas de oxígeno diarias; no se encontró asociación estadística entre las variables sociodemográficas como, sexo, estrato y ocupación; con relación a los determinantes ambientales no se halló asociación al relacionarlos con cada una de las dimensiones de calidad de vida, sin embargo, al evaluar la dimensión actividades se evidenció asociación estadísticamente significativa con las variables, grado de la enfermedad, tipo de inhalador, horas de oxígeno diarias, vacuna anti-influenza, uso de inhaladores, cámara espaciadora y oxígeno (Valores p <0,05) (Tabla 2).

Al correr al modelo de regresión logística para identificar las variables relacionadas con la variabilidad de las dimensiones se encontró que según aspectos sociodemográficos, al relacionar calidad de vida con etnia, se halló que la variables no es un factor de riesgo para determinar las alteraciones percibidas por los adultos en su calidad de vida (OR crudo: 8,00 IC 0,809-79,062), sin embargo, al ajustar calidad de vida con las variables sociodemográficas como sexo, nivel de escolaridad, estrato, estado civil, ocupación, vive con, consumo de cigarrillo y edad, la variable etnia se torna como un riesgo al indicar que quienes señalaron ser mestizos tienen 33,47 veces más riesgo de percibir altas o máximas alteraciones en su calidad de vida (OR ajust: 33,47 IC 1,541$726,870)$.

Al evaluar calidad de vida y los aspectos relacionados con la enfermedad, quienes tienen la enfermedad en un estado grave tienen 3,059 veces más riesgo de tener altas o máximas alteraciones en su calidad de vida en comparación con quienes tienen la enfermedad en un grado leve (OR crudo: 3,059 IC 1,140-8,207); sin embargo, al ajustar calidad de vida con las variables uso de inhaladores, tipo de inhalador, uso de cámara espaciadora, uso de oxígeno y horas diarias de uso, esta deja de ser de riesgo.

De forma similar, se halló que el usar inhaladores aporta 16,94 veces más riesgo de tener alteraciones altas o máximas en la calidad de vida (OR crudo: 16,94 IC 2,031-141,276); al ajustar por otras variables relacionadas con la enfermedad esta deja de ser de riesgo. El uso de cámara espaciadora también influye en la variabilidad de la percepción de alteraciones en la calidad de vida de los pacientes 3,048 veces más en comparaciones con quienes no las usan; sin embargo, al ajustar estos valores por las variables relacionadas con la enfermedad pierde su fuerza. El uso de oxígeno en adultos con EPOC los condiciona a tener 3,492 veces más riesgo de percibir alteraciones altas o máximas en su calidad de vida (OR crudo: 3,492 IC 1,768-6,899), al ajustarla por las demás variables relacionadas con la enfermedad esta se incrementa con 6,387 veces más riesgo de percibir alteraciones altas o máximas en su calidad de vida en comparación con quienes no hacen uso del oxígeno.

En relación a la dimensión o subescala Síntomas, se encontró que quienes tienen la enfermedad en un estado muy grave poseen 3,062 veces más riesgo de tener alta o máxima frecuencia síntomas respiratorios en comparación con quienes tienen la enfermedad en un estado leve (OR crudo: 3,062 IC 1,327-7,069). Así mismo quienes utilizan inhalador tienen 9,63 veces más riesgo de presentar alta o máxima frecuencia de síntomas respiratorios en comparación con quienes no lo utilizan (OR crudo: 9,625 IC 1,159-79,960). 
Tabla 2. Asociaciones entre las subescalas del instrumento de calidad de vida Saint George de los pacientes con enfermedad pulmonar obstructiva crónica EPOC, según características sociodemográficas, determinantes ambientales y aspectos relacionadas con la enfermedad

\begin{tabular}{|c|c|c|c|c|c|c|}
\hline \multicolumn{3}{|c|}{$\begin{array}{l}\text { Sociodemográficos } \\
\end{array}$} & \multirow{2}{*}{$\begin{array}{l}\text { Ambientales } \\
\text { Variable IND }\end{array}$} & \multirow[b]{2}{*}{ Valor P } & \multicolumn{2}{|l|}{ Enfermedad } \\
\hline Var & ble IND & Valor P & & & Variable IND & Valor P \\
\hline \multirow{10}{*}{ 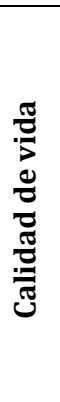 } & Tipo centro & 0,324 & Material vivienda & 0,78 & Grado enfermedad & 0,009 \\
\hline & Barrio & 0,005 & Otro cual & 0,09 & Utiliza inhaladores & 0,002 \\
\hline & Sexo & 0,822 & Servicios agua & const. & Tipo inhalador & 0,007 \\
\hline & Nivel escolaridad & 0,005 & Servicios saneamiento & const. & Cámara_espaciadora & 0,000 \\
\hline & Estrato & 0,148 & Servicios energía & const. & Utiliza oxígeno & 0,000 \\
\hline & Estado civil & 0,000 & Utiliza leña & 0,809 & Horas oxígeno & 0,352 \\
\hline & Etnia & 0,014 & Escenarios deportivos & 0,798 & Vacuna_influneza & 0,394 \\
\hline & Ocupación & 0,138 & Satisfecho & 0,33 & _- & _ \\
\hline & Tipo centro & 0,841 & Material vivienda & 0,947 & Grado enfermedad & 0,017 \\
\hline & Barrio & 0,153 & Otro cual & 0,552 & Utiliza inhaladores & 0,037 \\
\hline \multirow{11}{*}{ 苞 } & Sexo & 0,135 & Servicios agua & const. & Tipo inhalador & 0,17 \\
\hline & Nivel escolaridad & 0,308 & Servicios saneamiento & const. & Cámara_espaciadora & 0,067 \\
\hline & Estrato & 0,46 & Servicios energía & const. & Utiliza oxígeno & 0,171 \\
\hline & Estado civil & 0,641 & Utiliza leña & 0,888 & Horas oxígeno & 0,002 \\
\hline & Etnia & 0,085 & Escenarios deportivos & 0,374 & Vacuna_influneza & 0,945 \\
\hline & Ocupación & 0,48 & Satisfecho & 0,162 & - & _ \\
\hline & Vive_con & 0,156 & - & - & - & - \\
\hline & Consume cigarrillo & 0,741 & - & - & - & - \\
\hline & Tipo centro & 0,987 & Material vivienda & 0,525 & Grado enfermedad & 0,008 \\
\hline & Barrio & 0,706 & Otro cuál & 0,859 & Utiliza inhaladores & 0,000 \\
\hline & Sexo & 0,998 & Servicios agua & const. & Tipo inhalador & 0,003 \\
\hline \multirow{9}{*}{ 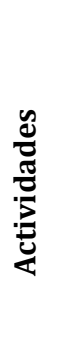 } & Nivel escolaridad & 0,087 & Servicios saneamiento & const. & Cámara_espaciadora & 0,000 \\
\hline & Estrato & 0,097 & Servicios energía & const. & Utiliza oxígeno & 0,000 \\
\hline & Estado civil & 0,015 & Utiliza leña & 0,986 & Horas oxígeno & 0,001 \\
\hline & Etnia & 0,063 & Escenarios deportivos & 0,191 & Vacuna_influneza & 0,049 \\
\hline & Ocupación & 0,638 & Satisfecho & 0,721 & _- & - \\
\hline & Vive_con & 0,775 & - & - & _- & - \\
\hline & Consume cigarrillo & 0,106 & - & - & _- & _ \\
\hline & Tipo centro & 0,542 & Material vivienda & 0,204 & Grado enfermedad & 0,471 \\
\hline & Barrio & 0,028 & Otro cual & 0,005 & Utiliza inhaladores & 0,017 \\
\hline \multirow{8}{*}{ 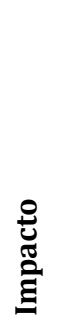 } & Sexo & 0,522 & Servicios agua & const. & Tipo inhalador & 0,327 \\
\hline & Nivel escolaridad & 0,096 & Servicios saneamiento & const. & Cámara espaciadora & 0,001 \\
\hline & Estrato & 0,352 & Servicios energía & const. & Utiliza oxígeno & 0,3 \\
\hline & Estado civil & 0,393 & Utiliza leña & 0,881 & Horas oxígeno & 0,556 \\
\hline & Etnia & 0,049 & Escenarios deportivos & 0,975 & Vacuna_influneza & 0,874 \\
\hline & Ocupación & 0,176 & Satisfecho & 0,296 & - & - \\
\hline & Vive_con & 0,106 & - & - & - & - \\
\hline & Consume cigarrillo & 0,084 & _- & _ & _ & _ \\
\hline
\end{tabular}

Los pacientes encuestados que utilizaban cámara espaciadora tienen 2,021 veces más riesgo de tener alta o máxima frecuencia de síntomas respiratorios en comparación con quienes indicaron no utilizar la cámara espaciadora (OR crudo: 2,021 IC 1,006-4,061). Los adultos que indicaron hacer uso de oxígeno tiene 1,942 veces más riesgo de percibir alta o máxima frecuencia de síntomas respiratorios en comparación con quienes no lo usan (OR crudo: 1,942 IC 1,0113,730 ). Sin embargo, al ajustar por las demás variables relacionadas con la enfermedad, ninguna de las consideradas anteriormente mostro ser de riesgo.

Según la subescala actividades, los adultos que indicaron utilizar inhalador tienen 13,333 veces más riesgo de presentar altas o máximas 
limitaciones producidas por la disnea en comparación con quienes no utilizan inhalador (OR crudo: 13,33 IC 2,949-60,279). Al ajustar por las demás variables relacionadas con la enfermedad en esta dimensión, esta condición deja de ser de riesgo; aquellos pacientes que utilizan cámara espaciadora tienen 3,63 veces más riesgo de presentar altas o máximas limitaciones producidas por la disnea (OR crudo: 3,63 IC 1,369-9,473), de igual forma al ajustarla no se torna de riesgo. Quienes indicaron utilizar oxígeno tienen 8,327 veces más riesgo de percibir altas o máximas limitaciones a causa de la disnea (OR crudo: 8,327 IC 3,197-21,689), esta variable al ser ajustada por las demás relacionadas con la enfermedad en esta dimensión, disminuye su riesgo a 7,17 veces en comparación con quienes no usan oxígeno.

Con respecto a la subescala Impacto, los pacientes que utilizan cámara espaciadora tienen 4 veces más riesgo de tener alta o máxima presencia de problemas de funcionamiento social y psicológico producidos por la enfermedad respiratoria (OR crudo: 4,00 IC 1,883-8,502), dicha variable al ajustarla por las demás relacionadas con la enfermedad aumenta su riesgo a 5 veces más en comparación con quienes no la usan (OR ajust: 5,001 IC 2,057-12,159).

\section{Discusión}

En un estudio desarrollado por Caballero et al. ${ }^{15}$, en 5 ciudades colombianas (Bogotá, Cali, Barranquilla, Bucaramanga y Medellín) la prevalencia encontrada fue de 8,9\%, con mayor predominio en el género masculino y mayores de 60 años; respecto a la posición geográfica, la prevalencia de EPOC en la ciudad de Medellín fue la más alta del país, con un 13,5\%; estos datos son similares a los hallados en el presente estudio donde los pacientes encuestados tenían entre 65 y 99 años de edad, el 50\% de las pacientes tenían 76 años o menos, y un promedio de edad de 77,08 años (DE $\pm 7,979)$.

Respecto al sexo se encontró que la prevalencia es mayor en mujeres $(57,54 \%)$, coincidiendo con estudios que demuestran que la prevalencia de
EPOC se está incrementando en todo el mundo, fundamentalmente asociada al aumento en las mujeres ${ }^{16}$.

En relación al nivel de escolaridad el 44,7\% tenían un nivel de formación básica primaria y solo el 10,6\% poseían educación básica secundaria, lo que se reflejó en las pocas oportunidades laborales que tuvieron estas personas, en comparación con un estudio desarrollado en la ciudad de Quito donde el nivel de educación observado fue en su mayor porcentaje primaria $43,8 \%$, seguido de secundaria $30 \%$ y superior $20 \%{ }^{17}$.

Respecto a la calidad de vida, se encontró que de forma general los adultos con EPOC, tienen puntuaciones superiores en la dimensión

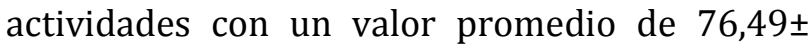
21,22 siendo esta la más afectada, esto indica que en mayor proporción los adultos tienen limitaciones para realizar ciertas actividades como caminar o desplazarse, al igual que la edad avanzada y al uso del oxígeno por largos periodos de tiempo de la gran mayoría de los pacientes, quienes incluso manifestaron sus dificultades para el desplazamiento dentro del hogar, dichas condiciones determinaron esta puntuación. El puntaje general promedio obtenido para calidad de vida con el SGRQ fue de 58,71 (DE $\pm 14,64$ ); para la dimensión que valoro el impacto fue de $50,49(\mathrm{DE} \pm 15,48)$ y para la dimensión que valoró la percepción de síntomas fue de 52,53 (DE \pm $60,02)$.

En la investigación desarrollada sobre la relación entre datos objetivos y calidad de vida percibida por pacientes con EPOC en Madrid, se hallaron resultados similares donde la puntuación total fue de 40,49 (DE $\pm 18,98$ ), para la dimensión que valoro el impacto el promedio fue de 32,86 (DE \pm 19,96); para síntomas fue de $34,76(\mathrm{DE} \pm 16,31)$ y para actividades de 56,68 (DE $\pm 23,72$ ); lo cual muestra que en esta investigación dicho promedio fue superior. En un estudio realizado en adultos mayores se encontró que la mayor afectación se tenía en la escala impacto lo cual se atribuían a la baja autoestima y a una percepción negativa en su calidad de vida ${ }^{18,19}$. 
En otro estudio realizado con pacientes con fibrosis quística a quienes también se les aplico el SGRQ, se encontró que a pesar de ser más jóvenes, la percepción de las alteraciones en su calidad de vida relacionada con la salud es más negativa al compararlos con una población pacientes con EPOC 20.

Adicionalmente, en la última década y sobre todo en los últimos 5 años, los resultados de un gran número de publicaciones han sugerido que existen otros factores ambientales de riesgo, además del tabaquismo, que se asocian con la EPOC; entre los más importantes están: la contaminación ambiental en el exterior e interior del hogar, la exposición laboral a polvos y humos, y un bajo nivel socioeconómico. En la literatura se indica que usualmente la EPOC es una patología de alta prevalencia en los estratos socioeconómicos menos favorecidos 21,22, datos similares a los hallados en este estudio donde la gran mayoría de pacientes corresponden a nivel socioeconómico bajo.

Según el estudio sobre prevalencia de la enfermedad pulmonar obstructiva crónica y sus principales factores de riesgo se encontró que respecto a la exposición a carbón para cocinar o calefacción en la casa estuvo presente en 13,8\% de los sujetos evaluados, mientras que la exposición al humo de leña fue de 56,5\%, para el caso de este estudio se encontró en proporción no muy distante que el 43,6\% manifestó haber cocinado sus alimentos en algún momento de su vida con leña ${ }^{23}$.

\section{Conclusiones}

Las personas encuestadas tenían un promedio de edad entre 65 y 99 años de edad, de estrato socioeconómico bajo y nivel de escolaridad, de raza mestiza y la gran mayoría habían sido consumidores de cigarrillo; además una gran proporción de adultos habían cocinado con leña y presentaban la enfermedad en estado muy grave; adicionalmente hacían uso del oxígeno varias horas al día y en ocasiones del inhalador. Los pacientes que tienen la enfermedad en un estado más severo, es mayor su percepción frente las alteraciones en su calidad de vida.

En cuanto a la calidad de vida, los valores obtenidos sugieren mayor afectación en la dimensión actividad ya que posiblemente se trataba de personas muy adultas y tenían grandes dificultades para desplazarse, bajo esta línea, le fue seguida por la dimensión impacto $y$ posteriormente los síntomas. Las variables que se correlacionan con mayor compromiso en la calidad de vida son: grado de la enfermedad, tipo de inhalador, utiliza oxígeno, número de horas de uso de oxígeno, uso de cámara espaciadora, nivel de escolaridad bajo, estrato socioeconómico $1 \mathrm{y}$ mestizo.

En general la calidad de vida se evidenció más afectada en los hombres que en las mujeres pese, a que en este estudio se encontraron mayor cantidad de mujeres con este diagnóstico, lo que corrobora un aumento del EPOC en los últimos años a expensas del sexo femenino, lo cual puede atribuirse principalmente a los estilos de vida.

La dimensión actividades mostro una afectación mayor en caso del sexo masculino donde se demostró mayores limitaciones debido a la dificultad respiratoria, además quienes tenían la enfermedad en un estado muy grave tenían mayor afectación de esta dimensión; a nivel general se encontró que las personas encuestadas se ven impactadas en un bajo grado debido a este tipo de situaciones. Los hombres manifestaron menos presencia de problemas de funcionamiento social y psicológico producidos por el EPOC en comparación con las mujeres.

Respecto a la dimensión síntomas se halló que quienes habían sido consumidores de cigarrillo o consumían actualmente, además de estar ubicados en el estrato socioeconómico 1 , se ven afectados por la presencia de síntomas respiratorios, como tos, pitos o silbidos en los pulmones, lo que les genera mayor dificultad respiratoria y alteración de su calidad de vida.

Al relacionar los resultados obtenidos en la medición de calidad de vida se encontró que existe asociación estadísticamente significativa entre calidad de vida total del instrumento 
específico para EPOC Saint George y las variables sociodemográficas como nivel de escolaridad, barrio, etnia y las variables relacionadas con la enfermedad como grado de la enfermedad, utiliza inhaladores, tipo de inhalador, cámara espaciadora y uso de oxígeno, pero al relacionarla con los determinantes ambientales no se encontró asociación alguna, es decir que existe una asociación directa con los aspectos relacionados con la enfermedad y la calidad de vida relacionada con salud de los pacientes con EPOC.

Conflicto de Intereses. Los autores declaran que no existen conflictos de intereses.

\section{Referencias}

1. Celli B, MacNee W, ATS/ERS Task Force. Standards for the diagnosis and treatment of patients with COPD: a summary of the ATS/ERS position paper. Eur Respir J 2004;23:932-46.

2. Colombia. Ministerio de Salud y Protección Social. Enfermedad pulmonar obstructiva crónica (EPOC). [Consultado 2016 junio 24] Disponible en https://www.minsalud.gov.co/salud/publica/PENT/Pa ginas/Enfermedad-pulmonar-obstructiva-cronica.aspx

3. Lin YX, Xu WN, Liang LR, Pang BS, Nie XH, Zhang J, et al. The cross-sectional and longitudinal association of the BODE index with quality of life in patients with chronic obstructive pulmonary disease. Chin Med J (Engl). 2009; 122:2939-44.

4. Araujo ZT, Holanda G. Does the BODE index correlate with quality of life in patients with COPD? J Bras Pneumol. 2010;36:447-52.

5. Buist AS, McBurnie MA, Wollmer WM, Gillespie S, Burney P, Mannino DM, et al. International variation in the prevalence of COPD. Lancet. 2007;370:741-50.

6. Braveman PA, Cubbin C, Egerter S, Williams DR. The social determinants of health: coming of age. Annu. Rev. Public Health. 2011;32:381-98.

7. Organización Mundial de la Salud. Comisión sobre determinantes sociales de la salud. [Consultado 2016 junio 24] Disponible en: http://www.who.int/social_determinants/resources/c sdh_brochure_spanish.pdf?ua=1.

8. Organización Mundial de la Salud. Cada año mueren 12,6 millones de personas a causa de la insalubridad del medio ambiente. [Consultado 2016 junio 27] Disponible en:

http://www.who.int/mediacentre/news/releases/201 6/deaths-attributable-to-unhealthy-environments/es/

9. Galvão L, Finkelman J, Henao S. Determinantes ambientales y sociales de la salud [consultado 2016 junio 27] Disponible en: http://www.paho.org/blogs/paltex/wp
content/uploads/2010/11/Finkelman_Cap00_semifina l_27.09.10_bis.pdf.

10. Soto, M, Failde, I. Calidad de vida relacionada con la salud como medida de resultados en pacientes con cardiopatía isquémica. Rev. Soc. Esp. Dolor. 2004;11(8).

11. Hinojosa, F. Enfermedad pulmonar obstructiva crónica (EPOC). Acta méd. Peruana. 2009;26(4).

12. Stahl E, Jansson SA, Jonsson AC, Svensson K, Lundbäck B, Andersson F. Health-related quality of life, utility, and productivity outcomes instruments: ease of completion by subjects with COPD. Health and Quality of Life Outcomes. 2003;1:18.

13. Menezes AM, Perez-Padilla R, Jardim JR, Muiño A, Lopez MV, Valdivia G, et al. Chronic obstructive pulmonary disease in five Latin American cities (the PLATINO study): a prevalence study. Lancet. 2005;366 (9500):1875-81.

14. Ferrer M, Villasante C, Alonso J, Sobradillo V, Gabriel R, Vilagut $\mathrm{G}$, et al. Interpretation of quality of life scores from the St Georges Respiratory Questionnaire Eur Respir J. 2002;19:405-413.

15. Caballero AS, Torres C, Maldonado D, Jaramillo C, Guevara D. Prevalencia de la enfermedad pulmonar oclusiva crónica en cinco ciudades colombianas. Trabajo presentado en X Congreso Colombiano de Neumología y Cirugía de tórax. Cartagena. 2003.

16. Torres J, Ciro C. EPOC en la mujer. Arch Bronconeumol. 2010;46(Supl 3):23-27.

17. Rivadeneira MF. Validación del cuestionario respiratorio St. George para evaluar calidad de vida en pacientes ecuatorianos con EPOC. Rev Cuid. 2015;6(1): 882-91.

18. Aibar Arregui MA, Laborda Ezquerra K, Conget López F. Relación entre datos objetivos y calidad de vida percibida por el paciente con EPOC. An Med Interna (Madrid) 2007; 24: 473-477.

19. Incalzi R, Bellied V, Catalana F, Scochilone N, Imperially C, Maggi S, et al. Evaluation of health outcomes in elderly patients with asthma and COPD using disease-specific and generis instruments. The Salute Respiratoria nell'Anziano (Sa.R.A.) Study. Chest. 2001;120(3):73442.

20. Ferrer M, Villasante C, Alonso J, Sobradillo V, Gabriel R, Vilagut $\mathrm{G}$, et al. Interpretation of quality of life scores from the St George's Respiratory Questionnaire. Eur Respir J. 2002;19:405-13.

21. Calle-Rubio M, Rodríguez-Hermosa JL, Álvarez-Sala Walther JL. EPOC en individuos no fumadores Arch Bronconeumol. 2010;46(Supl 4):16-21.

22. Piñol-Moreso L. Eficacia de la fisioterapia respiratoria estandarizada en atención primaria en los pacientes con EPOC moderada y su evaluación económica. (Tesis doctoral). Universidad de Cataluña, Cataluña, España. 2009. Disponible en: http://www. tdx.cat/bitstream/handle/10803/9356/Tesis_ Montse_Ingles.pdf;jsessionid=8D92C392080D E5BF6E2EF0A15CCC3535.tdx2? sequence=1.

23. Muiño A, López M, Menezes A. Prevalencia de la enfermedad pulmonar obstructiva crónica y sus principales factores de riesgo: proyecto PLATINO en Montevideo. Rev Med Uruguay. 2005;21:37-48. 